\title{
EVALUACIÓN DE LA CONFIABILIDAD DE UNA PRUEBA PARA IDENTIFICAR HÁBITOS DE ESTUDIO EN JÓVENES DE 14 A 20 AÑOS
}

EVALUATION OF THE RELIABILITY OF A STUDY HABITS ASSESSMENT TOOL FOR STUDENTS AGED BETWEEN 14 AND 20 YEARS OLD

\section{LUIS RAMÍREZ VERA* \& JOSÉ PARRA FICA**}

\section{RESUMEN}

El presente estudio tiene por objetivo evaluar la confiabilidad de una Prueba de diagnóstico para identificar hábitos de estudio en jóvenes de 14 a 20 años, recientemente diseñada y dada a conocer. El estudio se desarrolló con 160 estudiantes de cuarto año de enseñanza media y primer año de educación superior, en la Región del Maule, Chile. El análisis estadístico arrojó una fiabilidad por consistencia interna (Alpha de Cronbach) de 0.88. Se identifican tres áreas de influencia en los hábitos de estudio de jóvenes estudiantes; Ambiente y Recursos (AR) con un Alpha de 0.789, Voluntad y Responsabilidad (VR) Alpha 0.735 y finalmente Método y Trabajo (MT) con 0,688 como índice Alpha. Se concluye que el instrumento es confiable y que puede ser utilizado para el fin que anuncia su rótulo. Resulta recomendable que esta prueba se aplique, para complementar (o ser complemento), con otro tipo de evaluación de hábitos de estudio, de manera de contemplar más de un punto de vista que el que constituye la sola auto percepción de los estudiantes evaluados.

Palabras clave: hábitos de estudio, voluntad, método.

* Universidad Católica del Maule. Talca, Chile. Iramirez@ucm.cl

** Director Escuela Pedagogía General Básica. Universidad Católica del Maule. Talca, Chile. jparra@ ucm.cl 


\begin{abstract}
The purpose of the present study was to evaluate the reliability of a study habits assessment tool (In Spanish Prueba de diagnóstico para identificar hábitos de estudio en jóvenes de 14 a 20 años), which was recently designed by the authors. The participants in this study were 160 students, including senior high school and freshman college students, attending some educational institutions at the Maule Region in Chile. According to analyses, the internal consistency (Cronbach's Alpha) of the assessment tool was .88. Three factors that impact students' study habits were identified: Setting and Resources (SR) with an Alpha of .789; Willingness and Responsibility (WR) with an Alpha of .735; and Ethic Work (EW) with an Alpha of .688. The results of this study indicated that the study habits assessment tool (In Spanish Prueba de diagnóstico para identificar hábitos de estudio en jóvenes de 14 a 20 años) is reliable. Moreover, it is recommended that this assessment tool can be administered along with other study habits assessments to obtain a broader and deeper perspective on how high school and college students study.
\end{abstract}

Key words: Study habits, willingness, Ethic work.

\title{
1. INTRODUCCIÓN
}

El tema de los hábitos de estudio es longitudinal a toda la historia de la educación sistemática y también transversal a la realidad del currículo. Siempre fue, es y será necesario poner en vigor la intención y la acción de estudiar, si el propósito prioritario es aprender. El trabajo del estudiante es estudiar. A partir de ello, en la actualidad, se ha hablado de la profesionalización del estudio o del estudio como quehacer profesional (Castillo \& Polanco, 2005).

No obstante, también en el tiempo en que vivimos se ha impugnado, no sin argumentos, la asignación de tareas para la casa si el niño o el joven de enseñanza básica o secundaria está sometido a un régimen de jornada escolar completa.

Algo similar ocurre cuando, al considerar a las actuales generaciones de estudiantes como nativos digitales, puede entenderse equivocadamente que no requieren estudiar como siempre lo hicieron, habida cuenta que sus innegables habilidades les permiten -sin más- acceder a una información que está toda disponible y al alcance con nada más oprimir un signo en un teclado o en una pantalla.

Estudiar debe entenderse como una acción intencionada, organizada, sistemática y permanente en el tiempo, por el cual una persona -conforme a los objetivos que se han planteado en las materias o cursos que sigue- busca aprender para poder calificarse y promoverse (Ramírez, 2011). 
Del mismo modo, puede llevar a error limitar la necesidad de un estudio pertinente, especialmente en los años de escolaridad elemental y media, suponiendo que ello no resulta tan relevante en la educación superior, porque las circunstancias de mayor libertad que ésta exhibe obliga mucho menos a someterse a una disciplina de estudio controlado (Ramírez, 2017).

En la educación superior pueden percibirse evidentes falencias, como las dinámicas tantas veces impuestas del sólo cumplir en busca de la aprobación del curso (por sobre el estudiar para aprender) y, como consecuencia de ello, de una falta de noción de proceso formativo dotado de cierta inconciencia de la necesidad de apropiarse del conocimiento y la que se ha denominado, entre otras referencias, sindrome de la carpeta cerrada' (Ramírez, 2015).

El hábito asociado al estudio adquiere, entonces, una especial relevancia. Un comportamiento que recoge estrategias metodológicas y técnicas de trabajo para convertirse en puente que alcance estilos y maneras de estudiar y aprender, que lleven al niño o al joven a un adecuado desempeño escolar y/o académico.

Respecto de los estudios relativamente recientes disponibles fuera y dentro de Chile y que pudieran entenderse como antecedentes teóricos y empíricos, aunque no tratan de diseño y validación de nuevos instrumentos, sino de aplicación de pruebas ya existentes, es importante señalar que casi totalidad de ellos se han desarrollado con jóvenes de educación universitaria. Es el caso de las investigaciones en México de Hernández, Rodríguez \& Vargas (2012), en Colombia, de Torres, Tolosa, Urrea \& Monsalve (2009), de Acevedo, Torres \& Tirado (2015) y en España, de Capdevila \& Bellmunt (2016).

En el caso de Chile, está disponible el trabajo de Vidal, Gálvez \& Reyes (2009), el de Arán \& Ortega (2012) y los seminarios para optar al grado de licenciado en educación por la Universidad Católica del Maule de Castro, Guerrero \& Muñoz (2016), Llanos \& Quiroz (2016) y Ortiz, Ramírez, Salinas \& Venegas (2017). Cabe destacar que estos tres últimos hicieron aplicación, como ejercicio piloto, de la prueba de diagnóstico de cuya validación da cuenta el presente artículo.

Ahora bien, el elenco de instrumentos para la evaluación y medición de hábitos de estudio es, particularmente fuera de Chile, bastante nutrido. Entre ellos están, por ejemplo, el Inventario de hábitos de estudio de Wrenn, en 1970; el Cuestionario de hábitos de estudio, de Illueca, en 1972; el ACH-73 de

\footnotetext{
${ }^{1}$ Los estudiantes dicen curso aprobado, curso olvidado.
} 
Adelicio Caballero, en 1972 (Mateos, 2001) y el Inventario de hábitos de estudio de Bernstein, en 1997 (Vidal, Gálvez \& Reyes, 2009).

Del mismo modo, está disponible una edición revisada del año 2014 del Inventario de Hábitos de Estudio - IHE, de Pozar, originado en 1985 y, un Cuestionario de Hábitos y Técnicas de Estudio - CTHE en 2002 de Álvarez \& Fernández (Capdevila \& Bellmunt, 2016).

No obstante, la Prueba de Diagnóstico para Identificar Hábitos de Estudio diseñada durante el año 2017 por el profesor Luis Ramírez Vera, académico adjunto de la Universidad Católica del Maule, Talca-Chile y evaluada según lo informa esta publicación, puede hacer un aporte relativamente significativo si se tiene en cuenta que dentro del país herramientas de esta índole no están presente como subsidio importante para el trabajo educativo sistemático y, particularmente, en el amplio ámbito de la orientación educacional y vocacional. Respecto de los instrumentos generados fuera del país, es el más actualizado, no tan sólo por ser el más reciente en el tiempo sino, además, porque permite incorporar en sus tres áreas y 60 reactivos situaciones que consideran el quehacer del estudiante en el aula, frente a su profesor. Además, tratándose de una prueba diagnóstica, se contesta bajo una modalidad de autoevaluación (no es la observación externa que hacen padres o maestros) $y$, finalmente, permite establecer un perfil de hábitos de estudios simple y suficientemente informativo de la realidad de cada sujeto.

Se trata de una prueba (anexa al artículo) que considera 60 reactivos distribuidos en tres áreas, importantemente incidentes en la adquisición y preservación de hábitos de estudioy rotuladas como:Ambientey Recursos (AR20), para referirse a las condiciones externas que están relacionadas o inciden importantemente en el hábito de estudiar; Voluntad y Responsabilidad (VR20), asociada a actitudes y acciones que, independientemente del nivel de inteligencia clásico, permiten en el estudiante un menor o mayor desempeño exitoso en la escuela y Método y Trabajo (MT-20), ámbito que congrega formas, procedimientos o estrategias que el estudiante pone en práctica en su trabajo de estudiar. Cada uno de los reactivos presenta situaciones pertinentes en el hábito de estudiar, frente a las cuales el estudiante genera una respuesta de carácter dicotómica, escogiendo aquella situación que corresponde a su realidad de estudiante y dejando, simplemente, sin contestar las que no lo representen. A partir de las respuestas es posible levantar un perfil de hábitos de estudio con una escala de valoración que arroja una evaluación para cada una de las tres áreas y también una de carácter general. La escala de valoración considera los siguientes niveles: raras veces o muy insatisfactorio, 
escasas veces o insatisfactorio, algunas veces o suficiente, la mayor parte de las veces o satisfactoria y siempre o muy satisfactorio.

\section{MÉTODO}

\section{Enfoque y diseño}

El trabajo presentado en este artículo tiene por objetivo:

a) Verificar la consistencia interna del instrumento que permite identificar los hábitos de estudio en jóvenes entre 14 a 20 años, usando como técnica de consistencia interna el coeficiente Alfa de Cronbach.

b) Indagar la reproductividad de la prueba hábitos de estudio en jóvenes entre 14 a 20 años, usando la técnica de mitades partidas.

En coherencia con el propósito de la investigación, se ha optado por un enfoque cuantitativo de diseño descriptivo.

\section{Población y Muestra}

El estudio fue desarrollado en dos centros educativos de la ciudad de Talca, región del Maule, Chile. Uno de ellos de enseñanza secundaria, de modalidad humanístico-científica y de carácter particular subvencionado. Con una matrícula escolar total de alrededor de 1100 estudiantes, distribuidos desde primer año de enseñanza básica a cuarto año de enseñanza media y una trayectoria de 12 años. Participaron 89 (55.6\%) estudiantes de los tres cuartos años medios, según un muestreo de tipo no probabilístico, sujeto a disponibilidad (Cardona, 2003). El otro, una institución de educación superior, presente en las ciudades de Talca y Curicó, también de la región del Maule, Chile, universidad perteneciente al Consejo de Rectores, acreditada ante las autoridades nacionales de educación y de identidad católica, con 25 años de trayectoria, sobre 300 académicos de jornada completa y más de 8.000 estudiantes de pregrado y postgrado. Se usó el mismo tipo de muestreo indicado anteriormente, tratándose ahora de estudiantes iniciales de dos carreras de pedagogía. Se evaluó a 71 (44.4\%) estudiantes, haciendo -entonces- un total de 160 sujetos investigados. De ellos 84 (52.5\%) mujeres y 76 (47.5\%) hombres, con edades que oscilaron entre los 17 y los 22 años. 


\section{Variables e instrumento}

Una variable es una propiedad que puede cambiar y cuya variación es susceptible de medición y observación (Hernández-Sampieri, Fernández-Collado \& Baptista-Lucio, 2014). Estas propiedades son esencialmente valiosas en investigación cuando pueden relacionarse unas con otras. En este caso, en que no se trata de una investigación de carácter correlacional, se aporta una definición conceptual y operativa de la variable hábito de estudio.

Conceptualmente, el hábito corresponde a un comportamiento ciertamente aprendido y que debido a su reiteración termina integrándose a la conducta (habitual) del individuo. La reiteración ordenada, consciente y hasta debidamente justificada, va haciendo el hábito el que, no obstante, en algún momento en el tiempo, dada su práctica reiterada, puede llegar a hacerse automático. Sumado a esto, el hábito de estudio está definido en función de su propósito más relevante, esto es aprender. No obstante, es posible reconocer hábitos de estudio pertinentes, que favorecen efectivamente este aprendizaje y no pertinentes aquellos que no consiguen ese objetivo y o propósito (Ramírez, 2011).

Operacionalmente, el hábito de estudio se define por la puntuación obtenida en cada uno de los 20 reactivos que configuran las tres áreas que componen el instrumento, utilizando una escala de 60 reactivos de carácter dicotómico, es decir, ausencia o presencia del hábito. En este caso, el estudiante indica si el hábito (siempre pertinente) corresponde o no a lo que es su forma de enfrentar el estudio. La puntuación de cada una de las tres áreas de la prueba de diagnóstico se obtiene de la suma de sus 20 reactivos, por lo tanto, los valores oscilan entre 0 y 20, siendo ésta la puntuación más alta.

Tratándose de un instrumento nuevo, este estudio consideró, además, las variables; género, nivel de enseñanza y edad como elementos que permitieran caracterizar de mejor manera la muestra. Conforme ellas, en futuras aplicaciones, podría hacerse hallazgos que muestren mejores o peores desempeños y la incidencia que la condición de edad y - sobre todo- nivel de enseñanza, pudieran tener. En aplicación de realidades particulares (un curso o un nivel de enseñanza en una escuela, por ejemplo) puede también verificarse, en cuanto a género, las diferencias estadísticas que puedan darse. Las tablas que a continuación se presentan permiten visualizar estas variables de una manera más clara y detallada. 
Tabla 1

Género de los participantes.

\begin{tabular}{lcc} 
Variable & F & $\%$ \\
\hline Masculino & 76 & 47,5 \\
\hline Femenino & 84 & 52,5 \\
\hline Total & 160 & 100 \\
\hline
\end{tabular}

Fuente: elaboración propia.

En la Tabla 1 se puede observar que 76 son del género masculino lo que equivale al 47,5\%, de igual forma se registra que 84 sujetos son de género femenino correspondiendo al 52,5\% de la muestra.

Tabla 2

Nivel de enseñanza.

\begin{tabular}{lcc} 
Variable & $\boldsymbol{F}$ & $\%$ \\
\hline Enseñanza Media & 89 & 55,6 \\
\hline Universitario & 71 & 44,4 \\
\hline Total & 160 & 100 \\
\hline
\end{tabular}

Fuente: elaboración propia.

Una segunda variable considerada se informa en la Tabla 2 (Nivel de enseñanza). Allí se registra lo declarado por los sujetos incluidos en el estudio y se da cuenta que el 55,6\% indican estar cursando la enseñanza media o secundaria lo que corresponde a 89 jóvenes, mientras que 71 estudiantes correspondientes al 44,4\%, declaran cursar estudios universitarios en primer año.

Finalmente, la Tabla 3 (Edad), comunica la tercera variable a considerar que es la edad de los participantes. Esta se dividió en dos intervalos, el primero de ellos considera a los estudiantes cuyas edades fluctúan entre los 16 y 18 años. Este grupo lo conforman 90 alumnos equivalentes al 56,3\%, los restantes 70 alumnos se sitúan en el grupo etario comprendido entre los 19 y 22 años de vida, siendo el 44,7\% de los encuestados. 
Tabla 3

Edad.

\begin{tabular}{ccc} 
Variable & $\boldsymbol{F}$ & $\%$ \\
\hline $16-18$ & 90 & 56,3 \\
\hline $19-22$ & 70 & 44,7 \\
\hline Total & 160 & 100 \\
\hline
\end{tabular}

Fuente: elaboración propia.

La prueba de diagnóstico para identificar hábitos de estudio (2017) se construye en función de tres áreas de influencia, anteriormente descritas. Consta de 60 reactivos que se dividen en tres áreas a estudiar (AR - VR - MT) correspondiendo 20 a cada una de ellas, tal como lo muestra la Tabla 4.

Tabla 4

Reactivos y Áreas de influencia.

\begin{tabular}{lc}
\multicolumn{1}{c}{ Área } & \multicolumn{1}{c}{ Reactivos } \\
\hline $\begin{array}{l}\text { Ambiente y } \\
\text { Recursos }\end{array}$ & $1,4,7,10,13,16,19,22,25,28,31,34,37,40,43,46,49,52,55,58$ \\
\hline $\begin{array}{l}\text { Voluntad y } \\
\text { Responsabilidad }\end{array}$ & $2,5,8,17,14,17,20,23,26,29,32,35,38,41,44,47,50,53,56,59$ \\
\hline Método y Trabajo & $3,6,9,12,15,18,21,24,27,30,33,36,39,42,45,48,51,54,57,60$ \\
\hline
\end{tabular}

Fuente: elaboración propia.

\section{RESULTADOS}

La confiabilidad de un instrumento determinado es entendida, para efectos de este artículo, como el grado en que el mencionado instrumento, en nuestro caso, la Prueba de diagnóstico para identificar hábitos de estudio en jóvenes de 14 a 20 años, permite medir de manera consistente una muestra de la población definida, garantizando que está libre de errores.

Hernández-Sampieri (2003), citado en Oviedo \& Campo-Arias (2005) indican en su artículo Aproximaciones al uso del coeficiente Alfa de Cronbach, 
que el investigador Lee J. Cronbach en el año 1951, describe su coeficiente como el promedio de las correlaciones entre los reactivos que componen un determinado instrumento. De esta manera permite evaluar la magnitud en que los diferentes ítems están correlacionados.

Es esperable que un grupo de reactivos que pretenden medir un factor común, muestren un valor de Alfa de Cronbach lo más cercano a 1.0.

Dando cuenta del primer objetivo que nos hemos propuesto - "Verificar la consistencia interna del instrumento que permite identificar los hábitos de estudio en jóvenes entre 14 a 20 años, usando como técnica de consistencia interna el coeficiente Alfa de Cronbach"- se busca comprobar la consistencia del instrumento a través de una aproximación a la validación de constructo que consistió en la cuantificación de la correlación que existe entre los reactivos que la componen.

\section{Tabla 5}

Consistencia interna de la prueba para identificar hábitos de estudio en jóvenes de 14 a 20 años.

\section{Estadísticas de fiabilidad}

Alfa de Cronbach de Cronbach basada en
elementos estandarizados $\quad \mathrm{N}$ de elementos

\begin{tabular}{lll}
\hline, 887 &, 884 & 60 \\
\hline
\end{tabular}

Fuente: elaboración propia.

En la Tabla 5 se puede ver los resultados de la prueba de confiabilidad aplicada al instrumento en evaluación. Se consideraron 60 reactivos distribuidos en las tres áreas de influencia en los hábitos de estudio ya indicadas, las que los jóvenes identifican como asociadas o no a su propia realidad. El coeficiente se obtiene calculando la varianza de los reactivos con un valor de 0.884 que se encuentra dentro del límite de 0.7 a 0.9, que indica una buena consistencia interna para esta prueba. 
Tabla 6

Consistencia interna de la prueba para identificar hábitos de estudio en jóvenes de 14 a 20 años por área de influencia.

\begin{tabular}{cccc}
$\begin{array}{c}\text { Área de } \\
\text { influencia }\end{array}$ & Alfa de Cronbach & $\begin{array}{c}\text { Alfa de Cronbach } \\
\text { basada en } \\
\text { elementos } \\
\text { estandarizados }\end{array}$ & N de elementos \\
\hline $\begin{array}{c}\text { Ambiente y } \\
\text { Recursos }\end{array}$ & .789 & .788 & 20 \\
\hline $\begin{array}{l}\text { Voluntady } \\
\text { Responsabilidad }\end{array}$ & .735 & .732 & 20 \\
\hline \begin{tabular}{l} 
Método y Trabajo \\
\hline
\end{tabular} & .688 & .674 & 20 \\
\hline
\end{tabular}

Fuente: elaboración propia.

Al calcular el coeficiente considerando cada una de las áreas de influencia, los resultados que se muestran en la Tabla 6 indican que los 20 reactivos que buscan autoevaluar el Ambiente y Recursos, como área de influencia, reportan un alfa de .789. Los que miden el área Voluntad y Responsabilidad, un alfa de .735 y, finalmente, los 20 reactivos que buscan autoevaluar Método y Trabajo, un coeficiente alfa de .688. Todos ellos se ubican dentro o muy cercano al límite de 0.7 a 0.9 lo que indica una buena consistencia interna para cada uno de los componentes o áreas declaradas en la prueba aplicada.

Respecto del segundo objetivo propuesto - "Indagar la reproductividad de la prueba hábitos de estudio en jóvenes entre 14 a 20 años usando la técnica de mitades partidas" - el método de mitades partidas permite determinar la confiabilidad de una prueba y requiere de una única aplicación, a diferencia de otros métodos que necesitan más de una aplicación. Consiste en dividir la totalidad de los datos en dos mitades y las puntuaciones o resultados obtenidos por los alumnos a quienes se les aplicó el instrumento someterlas a comparación. Se puede indicar que una prueba es confiable, es decir que puede aplicarse a otros grupos de características similares, garantizando que mide lo que indica o se propone medir. En este caso permite identificar hábitos de estudio de jóvenes, cuando las puntuaciones obtenidas en 
ambos grupos o mitades están correlacionadas fuertemente, equiparando la cantidad de sujetos con puntuaciones similares en ambas mitades.

La confiabilidad se corrige de acuerdo a la cantidad de reactivos que se incluyan en el instrumento de medición, en nuestro caso, esta prueba de hábitos de estudio. Cuanto más reactivos, la confiabilidad aumenta. Consecuentemente, los supuestos básicos de la teoría de la medición al usar pruebas, parten del comportamiento aleatorio del error que al tomar una cantidad considerable de casos la correlación entre los puntajes verdaderos y los del error, se acerca a cero. De ahí lo importante de considerar 160 casos para esta aplicación (Namakforoosh, 2010).

\section{Tabla 7}

Confiabilidad, método mitades partidas.

\section{Estadísticas de fiabilidad}

\begin{tabular}{|c|c|c|c|}
\hline \multirow[t]{5}{*}{ Alfa de Cronbach } & \multirow[t]{2}{*}{ Parte 1} & Valor &, 750 \\
\hline & & $\mathrm{N}$ de elementos & $30 a$ \\
\hline & \multirow[t]{2}{*}{ Parte 2} & Valor & ,835 \\
\hline & & $\mathrm{N}$ de elementos & $30 b$ \\
\hline & \multicolumn{2}{|c|}{$\mathrm{N}$ total de elementos } & 60 \\
\hline Correlación entre formularios & & &, 769 \\
\hline \multirow{2}{*}{$\begin{array}{l}\text { Coeficiente de Spearman- } \\
\text { Brown }\end{array}$} & \multicolumn{2}{|c|}{ Longitud igual } & ,869 \\
\hline & \multicolumn{2}{|c|}{ Longitud desigual } & ,869 \\
\hline \multicolumn{3}{|c|}{ Coeficiente de dos mitades de Guttman } & ,849 \\
\hline
\end{tabular}

Fuente: elaboración propia.

La confiabilidad entendida como la precisión y exactitud de un instrumento, llamada también homogeneidad o representatividad, se explica como el grado en que se obtienen respuestas homogéneas a diferentes reactivos sobre un mismo concepto o dimensión, en nuestro caso, los hábitos de estudio de jóvenes estudiantes. 
El método de mitades partidas permite, con una única aplicación, y la división del total de reactivos en dos mitades, comparar las puntuaciones obtenidas.

La prueba será confiable si se encuentra que las puntuaciones de ambas mitades están fuertemente correlacionadas. Como es posible observar en la Tabla 7, el coeficiente de Sperman-Brown para longitudes iguales es de 0,869 lo que nos indica que hay una alta confiabilidad, al ser el coeficiente muy cercano al 0,9. Esto da cuenta que entre la parte 1 y parte 2 del instrumento existe una fuerte correlación. Ambas partes son muy similares para medir los hábitos de estudio en jóvenes estudiantes, por lo tanto, altamente confiable.

\section{DISCUSIÓN}

Esta investigación tuvo como propósito evaluar la confiabilidad de la Prueba de diagnóstico para identificar hábitos de estudio en jóvenes de 14 a 20 años.

El éxito académico puede ser predicho o anticipado con un alto grado de certeza al identificar sujetos con hábitos de estudio instalados y permanentes en el tiempo, variables como espacio temporal dedicado al estudio y regularidad de este trabajo o actividad académica. En este caso, se pretendió evaluar el comportamiento de este instrumento para poner a disposición del mundo académico una prueba que permita apoyar el trabajo del profesor y sobre todo para que los jóvenes puedan autoevaluarse y fortalecer aquellas áreas descendidas, manteniendo las que tengan una buena representatividad en ellos.

El instrumento en su conjunto evidencia un nivel de confiabilidad interna alto, con un Alfa de Cronbach de .884 .

Almedir por Áreas de Influencia los resultadossonsimilares, concoeficientes .789 para Ambiente y Recursos; 735 para Voluntad y Responsabilidad y, finalmente, un 688 para Método y Trabajo, todos coeficientes que dan cuenta de una consistencia o grado de homogeneidad fuerte confirmando que el instrumento garantiza respuestas homogéneas al aplicar distintos reactivos sobre un mismo concepto, en este caso hábitos de estudio.

No obstante, una posible inferencia que es necesario señalar respecto al área de más baja puntuación -Método y Trabajo (.688)- tenga relación con el carácter de reactivos que representan un área de lenguaje menos coloquial 
y personal, y evidentemente más técnico en lo educativo y particularmente en lo pedagógico.

Finalmente, como una manera de verificar si el instrumento se puede reproducir, se aplica el método de mitades partidas que arroja un coeficiente .869, lo que corrobora una alta confiabilidad en el instrumento en estudio.

No se tenía disponible en Chile un instrumento de estas características, por lo cual es difícil hacer comparaciones con trabajos precedentes, al menos, relativamente recientes. El levantamiento de esta prueba de diagnóstico surge de la necesidad de servir, principalmente, en tres planos: su aplicación en realidades particulares, como estudiantes o grupos curso que requieran verificar de una manera técnica el estado de sus hábitos de estudio, para posteriormente tomar las decisiones de retroalimentación o enmienda que se requieran; además, contribuir al estudio y desarrollo de la investigación en el área de la orientación educacional, a partir del uso de un instrumento válido y confiable. Finalmente, tener disponible una herramienta académica para la formación de profesores y profesores jefes, en general y orientadores educacionales y vocacionales, en particular.

\section{CONCLUSIÓN}

Queda, entonces, a disposición de la comunidad educativa y académica, tanto nacional como extranjera, esta Prueba de diagnóstico para identificar hábitos de estudio, debidamente evaluada en cuanto a confiabilidad. Esta herramienta técnica permite conocer (incluso al estilo de una autoevaluación), el estado de la situación o conductas de entrada de cada joven (en un rango etario aproximado de 14 a 20 años) frente a reactivos distribuidos en tres áreas, que obedecen a tres categorías: Ambiente y Recursos, Voluntad y Responsabilidad y Método y Trabajo. La expectativa es que se transforme en un instrumento más como subsidio a la sistemática tarea de estudiar y pueda complementar o ser complementada por otros empeños académicos y resultados insertos en esta misma área disciplinar del conocimiento. Las proyecciones pueden ser varias, por lo pronto, el estudio que pueda correlacionar los resultados de una prueba como esta con el rendimiento escolar o desempeño académico que los jóvenes que la rindan puedan alcanzar.

Como limitaciones del presente estudio, aun los resultados obtenidos de 160 sujetos de entre 17 y 22 años, considerando el último nivel de enseñanza media y primer año de educación superior, podría señalarse -en primer lugar- que pudiera ser ésta una muestra todavía reducida. En segundo 
término, la no consideración de todo el rango etario al que está destinado el instrumento (desde 14 años, hasta 20). Sería importante verificar como funciona con jóvenes estudiantes, por ejemplo, de octavo año básico (14 años) o, segundo o tercero medio (con edades promedio de 16 y 17 años). Así mismo, otra limitación que debería subsanarse en el futuro, tiene que ver con el procedimiento de muestreo escogido, resultando recomendable incluir muestreos aleatorios con el fin de obtener una mayor generalización de los resultados.

\section{REFERENCIAS BIBLIOGRÁFICAS}

Acevedo, D., Torres, J. D., \& Tirado, D. F. (2015). Análisis de los hábitos de estudio y motivación para el aprendizaje a distancia en alumnos de Ingeniería de Sistemas de la Universidad de Cartagena (Colombia). Formación Universitaria, 8(5), 59-66. DOI: https://dx.doi.org/10.4067/ s0718-50062015000500007.

Arán, M., \& Ortega, M. (2012). Enfoques de aprendizaje y hábitos de estudio en estudiantes universitarios de primer año de tres carreras de la Universidad Mayor de Temuco, Chile 2011. Revista Educativa Hekademos, 5(11), 37-46.

Capdevila, A., \& Bellmunt, H. (2016). Importancia de los hábitos de estudio en el rendimiento académico del adolescente: diferencias por género. Educatio Siglo XXI, 34(1), 157-172.

Cardona, M. C. (2003). Introducción a los métodos de investigación en educación. Madrid: EOS.

Castillo, S., \& Polanco, L. (2005). Enseña a estudiar...aprende a aprender. Didáctica del estudio. Madrid: Pearson - Prentice Hall.

Castro, J., Guerrero, A., \& Muñoz, E. (2016). Hábitos de estudio en alumnos de Educación Superior: el caso del ingreso 2016 en la carrera de Pedagogía en Ciencias con mención (Seminario de grado y Título de profesor), Universidad Católica del Maule, Talca, Chile.

Hernández, C., Rodríguez, N., \& Vargas, A. (2012). Los hábitos de estudio y motivación para el aprendizaje de los alumnos en tres carreras de ingeniería. Revista de la Educación Superior, 47(163), 67-87. 
Hernández-Sampieri, R., Fernández-Collado, C., \& Baptista-Lucio, P. (2014). Metodología de la investigación (6a Ed.). México: McGraw-Hill/Interamericana.

Llanos, F., \& Quiroz, T. (2016). Verificación de hábitos de estudio en alumnos iniciales de enseñanza terciaria: el caso de Pedagogía en Matemática y Computación en la Universidad Católica del Maule (Seminario de grado y Título de profesor), Universidad Católica del Maule, Talca, chile.

Mateos, F. (2001). Estudio de las técnicas de estudio en adultos, Exphoros, 3, 133-146.

Namakforoosh, M. (2010). Metodología de la investigación. México: Limusa

Ortiz, M., Ramírez, L., Salinas, J., \& Venegas, L. (2017). Hábitos de estudio en estudiantes de Educación Superior: el caso del ingreso 2017 de la carrera de Pedagogía en Lengua Castellana y Comunicación, en la Universidad Católica del Maule (Seminario de grado y Título de profesor), Universidad Católica del Maule, Talca, Chile.

Oviedo, H. C., \& Campo-Arias, A. (2005). Aproximación al uso del coeficiente alfa de Cronbach. Revista Colombiana Psiquiatría, 34(4), 572-580.

Ramírez, L. (2011). Aprender y descubrir: breves y elementales orientaciones metodológicas para el estudio y la indagación. Talca: Ediciones Universidad Católica Del Maule.

Ramírez, L. (2015). Formación de profesores: Esbozo de seis desafíos. UCMaule, 48, 103-109. Recuperado de http://revistaucmaule.ucm.cl/article/ view/25

Ramírez, L. (2017). Hazte el hábito: prueba de diagnóstico y breves orientaciones en torno al hábito de estudiar. Talca: Helena Ediciones.

Torres, M., Tolosa, I., Urrea, M., \& Monsalve, A. (2009). Hábitos de estudio versus fracaso académico. Revista de Educación, 33(2), 15-24.

Vidal, L., Gálvez, M., \& Reyes, L. (2009). Análisis de hábitos de estudio en alumnos de primer año de ingeniería civil agrícola. Formación Universitaria, 2(2), 27-33. 


\section{ANEXO}

\section{PRUEBA DE DIAGNÓSTICO RAMÍREZ VERA PARA IDENTIFICAR HÁBITOS DE ESTUDIO}

\section{Instrucciones}

Este es un breve instrumento que contiene 60 situaciones referidas a hábitos de estudio que pueden o no estar asociadas a la forma como Usted enfrenta esta importante responsabilidad. No hay en él respuestas buenas ni malas. Toda respuesta es buena si representa fielmente su propia realidad con el estudio. Lea con atención cada situación y marque en la hoja de respuestas aquellas que se verifiquen en Usted en su condición de estudiante. Las que no, déjelas sin contestar. Dispone de 15 minutos para responder.

1. Me preocupo de tener o conseguir libros y textos de apoyo a las materias de mis clases.

2. Asisto regularmente a mis clases en la escuela.

3. Contextualizo o relaciono con la realidad lo que vamos trabajando en clases, para así entenderlo mejor.

4. Uso y consulto efectivamente textos o material bibliográfico de apoyo a mis clases.

5. Hago un esfuerzo permanente por estar muy atento al desarrollo de la clase.

6. Me pregunto qué valor o significado tiene para mí lo que debo aprender.

7. Cuando no estoy en la escuela alterno, adecuadamente, el estudio con el tiempo de descanso.

8. Participo, activamente, en clases.

9. Tengo claro el objetivo y propósito de cada clase.

10. Tengo, efectivamente, estructurado un horario de estudio más allá de la clase, en la escuela o el hogar. 
11. Solicito apoyo a mis compañeros de clase, cuando lo necesito.

12. Consulto o pregunto por el objetivo de la clase si el profesor no lo declara.

13. Mi horario de estudio involucra un tiempo para revisar y ordenar los apuntes de las clases del día.

14. Acostumbro a llegar puntualmente a clases y a todos mis compromisos relacionados con mi condición de estudiante.

15. Pido al profesor la síntesis de la clase o las ideas fundamentales trabajadas en ella.

16. Mi horario de estudio involucra un tiempo para iniciar el desarrollo de tareas y trabajos, sin importar el tiempo de rendición o entrega.

17. Reviso, habitualmente, mi agenda de trabajo y horario de actividades del día siguiente.

18. Solicito al profesor tema u objetivo de la siguiente clase.

19. Mi horario de estudio involucra un tiempo para releer las últimas materias de las clases que tendré al día siguiente.

20. Preparo mi bolso, con útiles e implementos de trabajo, la tarde o noche anterior al día de clases.

21. Tomo notas y apuntes para dejar registro escrito de los aspectos más relevantes de la clase.

22. Estudio en un lugar adecuado.

23. Selecciono diarios, revistas impresas o virtuales, información o material asociados a lo que estoy estudiando en mis clases.

24. Conozco efectivamente cómo aprendo mejor.

25. Estudio en el momento del día adecuado.

26. Ordeno mis apuntes inmediatamente después de la clase para complementarlos.

27. Pregunto y planteo mis dudas al profesor.

28. Intento recrearme adecuadamente. 
29. Después de revisar y ordenar mis apuntes los sintetizo en fichas, esquemas o mapas conceptuales.

30. Asumo que materias o disciplinas diferentes tienen diferente formas de estudiar y aprender.

31. Intento alternar el descanso o recreación y el estudio en los tiempos naturalmente correspondientes.

32. Busco en mi diccionario (virtual o impreso) las palabras y conceptos que no entiendo o pido al profesor el significado de esas palabras.

33. Estudio mis materias adecuadamente distribuidas en el tiempo disponible, evitando dejar todo para el final.

34. Evito todo tipo de distracción al momento de estudiar.

35. Busco información más allá de lo entregado en la clase, revisando libros de texto o información en Internet.

36. Estudio metódica y sistemáticamente, evitando el desorden y la improvisación.

37. Evito estudiar en el momento del día en que me siento más cansado.

38. Soy capaz de perseverar en el estudio (mantenerme estudiando) aunque me inviten a otras actividades que no están relacionadas con él.

39. Estudio primero sol@, antes de reunirme con mi grupo de trabajo, para así poder aportar efectivamente al trabajo en equipo.

40. Mantengo un lugar de estudio en forma permanente.

41. Llevo mis apuntes y cuadernos al día.

42. Asumo mi estudio como un proceso continuo y no como un hecho aislado.

43. Dispongo de materiales de trabajo suficiente para desarrollar mi estudio.

44. Mi voluntad es estudiar para aprender y no solo para aprobar.

45. Leo de manera disciplinada, intentando entender lo que leo. 
46. Frecuento la biblioteca de mi escuela y hago uso de ella cuando la necesito.

47. Soy capaz de solicitar a mis amig@s que no me llamen ni me busquen durante el tiempo que dedico al estudio.

48. Desarrollo, efectivamente, ensayos y ejercicios en las materias en donde hay que realizarlos y resolverlos.

49. Logro que en mi casa colaboren con un ambiente propicio para desarrollar mis tareas y estudio.

50. Ante una tarea o problema complejo insisto y hago el mayor y mejor esfuerzo para resolverlo.

51. Intento diferenciar en un texto las ideas principales de las no tan importantes.

52. Ante un suceso inesperado que me impida estudiar en el horario programado, tengo otro plan (plan B) de recuperación de ese tiempo.

53. Intento desarrollar mis tareas y trabajos con mínimas normas de honestidad, tratando de evitar el "cortar y pegar" y la copia indiscriminada de textos (plagio).

54. Transformo el texto y las materias en preguntas y cuestionarios, para desarrollarlos después.

55. Comento y relato a los miembros de mi familia y a otras personas lo que estoy aprendiendo.

56. Mantengo una actitud responsable ante mis horarios de trabajo en la escuela, evitando los atrasos y ausencias.

57. Intento comprender más que solo memorizar lo que recibo en mis clases o lecturas.

58. Antes de instalarme a estudiar procuro disponer de todo lo que requiero, para así evitar interrupciones.

59. Llevo ordenadamente mi agenda y calendario de pruebas y trabajos.

60. Corrijo mis errores, especialmente en pruebas y trabajos en lo que me he equivocado. 


\section{PRUEBA DE DIAGNÓSTICO RAMÍREZ VERA PARA IDENTIFICAR HÁBITOS DE ESTUDIO}

Apellido paterno:

Apellido materno:

Nombres:

Edad: Fecha:

HOJA PARA LAS RESPUESTAS

Encierra en un círculo el número de las situaciones que escojas

\begin{tabular}{|c|c|c|c|}
\hline 1 & 2 & 3 & \\
\hline 4 & 5 & 6 & \\
\hline 7 & 8 & 9 & \\
\hline 10 & 11 & 12 & \\
\hline 13 & 14 & 15 & \\
\hline 16 & 17 & 18 & \\
\hline 19 & 20 & 21 & \\
\hline 22 & 23 & 24 & \\
\hline 25 & 26 & 27 & \\
\hline 28 & 29 & 30 & \\
\hline 31 & 32 & 33 & \\
\hline 34 & 35 & 36 & \\
\hline 37 & 38 & 39 & \\
\hline 40 & 41 & 42 & \\
\hline 43 & 44 & 45 & \\
\hline 46 & 47 & 48 & \\
\hline 49 & 50 & 51 & \\
\hline 52 & 53 & 54 & \\
\hline 55 & 56 & 57 & \\
\hline \multirow[t]{3}{*}{58} & 59 & 60 & \\
\hline & & & (suma) \\
\hline & & & (porcentaje) \\
\hline
\end{tabular}


PRUEBA DE DIAGNÓSTICO RAMÍREZ VERA PARA IDENTIFICAR HÁBITOS DE ESTUDIO

Apellido paterno:

Apellido materno:

Nombres:

Edad: Fecha:

PERFIL DE HÁBITOS DE ESTUDIO

\begin{tabular}{l}
\hline 20 \\
\hline 19 \\
\hline 18 \\
\hline 17 \\
\hline 16 \\
\hline 15 \\
\hline 14 \\
\hline 13 \\
\hline 12 \\
\hline 11 \\
\hline 10 \\
\hline 9 \\
\hline 8 \\
\hline 7 \\
\hline 6 \\
\hline 5 \\
\hline 4 \\
\hline 3 \\
\hline 1 \\
\hline 0 \\
\hline AR ( \%)
\end{tabular}

Puntaje total general:

\footnotetext{
Tablas de conversión de puntajes obtenidos:

1-4: raras veces / muy insatisfactorio.

5-8: escasas veces / insatisfactorio.

9-12: algunas veces / suficiente

13-16: la mayor parte de las veces / satisfactorio

17-20: siempre / muy satisfactorio.
}

\section{Puntaje total general:}

1-12: rara vez / muy insatisfactorio. 13-24: escasas veces / insatisfactorio 25-36: algunas veces / suficiente

37-48: la mayor parte de las veces / satisfactorio. 49-60: siempre / muy satisfactorio. 
Descripción - interpretación: 


\section{PRUEBA DE DIAGNÓSTICO RAMÍREZ VERA PARA IDENTIFICAR HÁBITOS DE ESTUDIO}

\section{INSTRUCCIONES PARA EL PROFESOR}

1. Proceda a sumar las respuestas de las columnas correspondientes a cada una de las tres áreas:

1 a 58: Ambiente y Recursos/AR

2 a 59: Voluntad y Responsabilidad/VR y 3 a 60: Método y Trabajo/MT

Registre el total de cada una de ellas en el espacio asignado al final. Si quiere traducir los totales de cada columna a porcentajes, considere que cada respuesta marcada corresponde en a un 5\%. Este porcentaje lo puede anotar también en el espacio indicado. Haga, además, el conteo del puntaje total general.

2. Para construir el perfil de hábitos de estudio, marque sobre cada columna el número de respuestas dadas y levante la barra correspondiente. Es posible, alternativamente, unir los puntos de cada columna, trazando la curva respectiva.

3. En tanto debería entregarse este perfil como resultado obtenido a cada estudiante interesado, puede agregar en la base el porcentaje de respuestas en cada columna (está también en la hoja de respuestas), el puntaje general y constatar, en las tablas de conversión, el concepto obtenido en cada caso.

4. Como orientaciones para describir o interpretar el resultado obtenido, cabe destacar que éste es un antecedente más en el trabajo exploratorio de los hábitos de estudio en el sujeto evaluado. No es posible pretender hacer ninguna decisión determinante, sin buscar información válida complementaria. Como cada uno de los reactivos corresponde a situaciones pertinentes de considerar en el acto de estudiar, estos mismos pueden ser útiles al intentar recomendar enmiendas, complementos o generación y asentamiento de hábitos.

5. En la proyección del estudio está la posibilidad intentar comprobar cómo se relacionan hábitos con desempeño y rendimiento en los estudiantes. 